\title{
Management and Prevention of Hyperkalemia in Diabetes
}

\author{
Anil K. Mandal* \\ Courtesy Clinical Professor of Medicine, University of Florida at Gainesville, Florida USA
}

Received: December 12, 2015, Accepted: March 08, 2016, Published: March 17, 2016

*Corresponding author: Anil K. Mandal, Mandal Diabetes Research Foundation, 800 Zeagler Drive Suite 510, Palatka, Fl 32177, Florida, USA, Fax: 1-(904) 823-1284; Email: amandal@med-spec.com

\begin{abstract}
Hyperkalemia of variable severity is common in patients with uncontrolled or untreated diabetes. Insulin therapy avert hyperkalemia by translocating potassium intracellularly. The main reason of hyperkalemia in diabetes is due to associated acute renal failure or chronic renal failure. Both these accompaniment are mainly due to use of angiotensin converting enzyme inhibitor (ACEI) or angiotensin receptor blocker (ARB) or a combination of both. Therefore treatment of diabetes with appropriate dose of insulin and avoidance of the use of ACEI/ARB therapy is the mainstay of prevention of hyperkalemia in diabetes.
\end{abstract}

\section{Introduction}

Hyperkalemia is not as common as hypokalemia. If all patients with acute and chronic renal failure are excluded, the incidence of hyperkalemia is insignificant. However, preponderant use of angiotensin converting enzyme inhibitor (ACEI) and angiotensin receptor blocker (ARB) in the management of diabetes mellitus (DM) as a renoprotective drug has escalated the incidence of hyperkalemia. In some instances, hyperkalemia develops acutely, but in most instances hyperkalemia is chronic. In most instances, hyperkalemia is asymptomatic and is found by routine laboratory testing. Here is an example to that effect.

\section{Case Report}

A 44 year old Spanish speaking man has had bilateral below knee amputation from gangrene associated with uncontrolled diabetes mellitus and possibly peripheral vascular disease. He went to a laboratory for routine blood testing on November 6 , 2015 before his office visit with the author. Upon questioning he denied any symptom like chest pain, palpitation or aching muscles. He denied receiving ACEI/ARB drugs, aldosterone antagonist or nonsteroidal anti-inflammatory drugs (NSAID). However, it should be kept in mind that people frequently take over the counter medications. One of the drugs is NSAID, ibuprofen and rarely patients will reveal that information. The results of his serum chemistry on November 6, 2015 at 1500 hours are shown in Table 1.

He was brought in to the emergency room and treated with $50 \%$ dextrose solution intravenously and 10 units of aspart insulin subcutaneously and calcium gluconate $10 \% 100 \mathrm{ml}$.
Thereafter he was admitted into hospital for further treatment. In the hospital, he was treated with sodium polystyrene sulfonate (kayexalate) 15g at 16:42 hours on November 6, 2015 and sodium bicarbonate infusion (50 meq sodium bicarbonate in a liter of normal saline). He received two additional doses of kayexalate 15 g each on November 7 at 6:19 am and 12:55 hours. He received an additional dose of calcium gluconate $1 \mathrm{~g}$ intravenously at 6:20 am on November $7^{\text {th }}$ and additional doses of $50 \%$ dextrose solution $50 \mathrm{ml}$ and aspart insulin 10 units subcutaneously on November 7 at 6:20 am. His home medications were resumed which consist of detemir insulin (Levemir $®$ ) 30 units after breakfast and dinner, aspart insulin (Novolog®) 18 units with each meal, hydroxyzine $50 \mathrm{mg}$ Po. BID, gabapentin $300 \mathrm{mg}$ Po BID, omeprazole $50 \mathrm{mg}$ PO daily, amlodipine $10 \mathrm{mg}$ BID, ferrous sulfate $350 \mathrm{mg}$ PO BID, and ambien $5 \mathrm{mg}$ at bedtime. Laboratory studies were done daily for diabetes control and electrolytes and acid base control. The results are shown in Table 2.

From these two tables, it is evident that patient developed severe hyperkalemia. The etiology of severe hyperkalemia is undetermined. He didn't take ACEI/ARB drugs; whether he took NSAID could not be confirmed. Nevertheless, his renal function recovered to normal level and hyperkalemia is corrected. His Co2 (Hco3) level was $18 \mathrm{mmol} / \mathrm{L}$. His Co2 increased to $22 \mathrm{mmol} / \mathrm{L}$ on November 8, 2015. His hemoglobin was $10.7 \mathrm{~g}$ on November 7 , 2015. Hemoglobin was not repeated. His diabetes is not under control.

\section{Etiology of Hyperkalemia}

Potassium in the food is absorbed in the gastrointestinal tract and filtered and completely excreted by the kidneys in those with

\begin{tabular}{|c|c|}
\hline \multicolumn{1}{|l|}{ Table 1: } \\
\hline BUN (6-19) mg/dl & Result \\
\hline Creatinine $(0.8-1.5) \mathrm{mg} / \mathrm{dl}$ & 71 \\
\hline eGFR (60-) ml/min & 1.70 \\
\hline Glucose (70-99) mg/dl & 47 \\
\hline Calcium (8.4-10.2) mg/dl & 356 \\
\hline Na $(135-150) \mathrm{mmol} / \mathrm{L}$ & 9.0 \\
\hline $\mathrm{K}(3.5-5) \mathrm{mmol} / \mathrm{L}$ & 130 \\
\hline \multicolumn{2}{r}{$\mathrm{CH}=$ critically high } \\
\hline
\end{tabular}




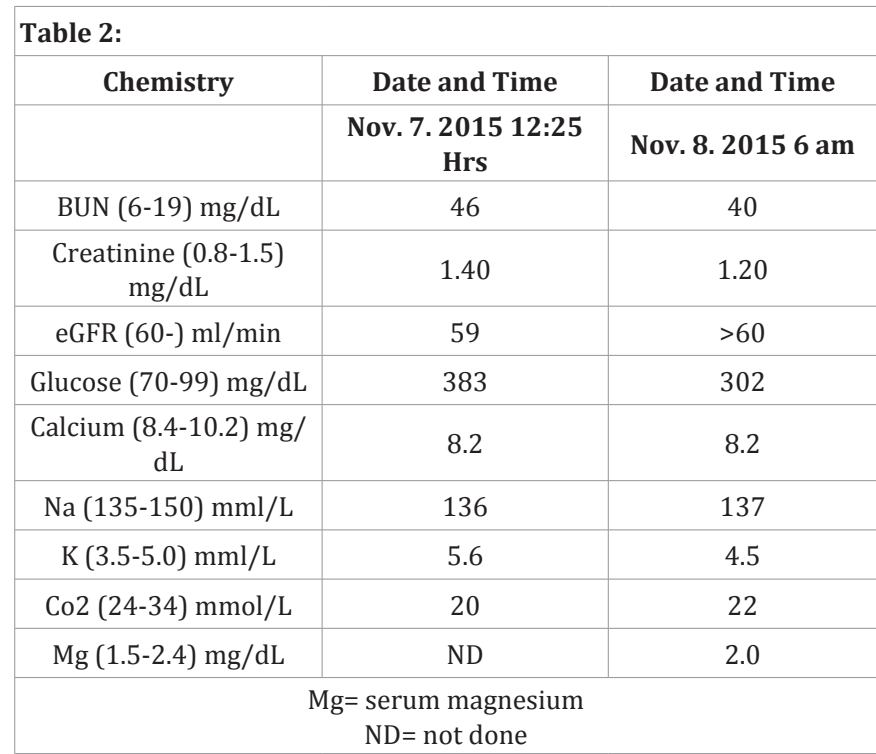

Table 3:

\begin{tabular}{|c|c|c|c|}
\hline \multicolumn{4}{|c|}{ Renal Function levels } \\
\hline & BUN(mg/dl) & $\begin{array}{c}\text { Serum creatinine } \\
(\mathrm{mg} / \mathrm{dl})\end{array}$ & eGFR $\mathrm{ml} / \mathrm{min}$ \\
\hline Upper Mean & 59.6 & 3.5 & 28.2 \\
\hline Lower Mean & 41.6 & 2.2 & 38.5 \\
\hline & $\begin{array}{r}\text { Total numl } \\
\text { Serum potas } \\
\text { Upper } \\
\text { Lower }\end{array}$ & $\begin{array}{l}\text { r of patients }=17 \\
\text { :F } 7: 10 \\
\text { dm levels }(\mathrm{mmol} / \mathrm{L}) \\
\text { nge: } 5.0 \text { to } 9.1 \\
\text { ean } 6.2 \\
\text { nge: } 4.9 \text { to } 6.0 \\
\text { ean } 5.2\end{array}$ & \\
\hline
\end{tabular}

normal $\mathrm{K}$ balance. Potassium excretion will be reduced when (1) filtration of potassium is decreased as a result of decrease of glomerular filtration rate (GFR); and (2) secretion of potassium is inhibited as a result of decreased mineralocorticoid activity. Potassium excretion is decreased in acute and chronic renal failure. Severe hyperkalemia occurs more commonly in acute renal failure than stable chronic renal failure.

\section{Drug Induced hyperkalemia}

Drug induced hyperkalemia is recognized as the most common cause of hyperkalemia

In clinical practice the most common group of drugs in that regard is renin-angiotensin converting enzyme (ACEI) drugs [1, 2]. Impaired renal function is the fundamental pathophysiologic basis of drug induced hyperkalemia. Here are some data to that effect. 119 patients were treated with ACEI; 46 of 119 (38 percent) developed hyperkalemia. Of these patients with hyperkalemia, 96 percent had chronic renal failure, 84 percent had diabetes and 90 percent had congestive heart failure (CHF) [1]. Here is an analysis of drug induced hyperkalemia from Putnam Community Medical Center, Palatka in 2008 with ICD code hyperkalemia (unpublished). The data is presented in Table 3.
Drug responsible for hyperkalemia in these 17 patients include lisinopril 4, spironolactone 4 , bactrim 2, naproxen 2 , losartan 2, telmisartan 1, potassium 1, enalapril 1, benazepril,1, irbesartan 1. Total number of patients who took ACEI/ARB were 10 (59 percent). Therefore ACEI/ARB group of drugs are the most common cause of hyperkalemia in hospitalized patients. Of these 17 patients 12 had CHF with renal insufficiency and 5 patients had hypertension and diabetes.

A discrete group of drugs that preferentially inhibit $\mathrm{K}$ secretion through the tubules resulting in hyperkalemia, they are

1. Potassium sparing drugs: spironolactone, triamterene, amiloride

\section{ACEI/ARB drugs}

3. Prostaglandin synthesis inhibitor

4. Trimmethoprim (Bactrim)

5. Beta Blockers

6. Other drugs: pentamidin, digitalis, calcineurin inhibitors such as cyclosporine or tacrolimus and succinyl chloride.

\section{Other Causes of Hyperkalemia}

Selective hypoaldosteronism: Hyperkalemia is the hallmark of selective hypoaldosteronism. Cardiac arrhythmias or cardiac arrest due to hyperkalemia often draws attention to the problem. Hypoaldosteronism may be idiopathic or secondary to hyporeninemia. The syndrome of hyperkalemia, hypoaldosteronism, and mild to moderate renal insufficiency is usually due to a hypoplastic juxtaglomerular apparatus seen in diabetic nephropathy or any form of tubulointerstitial nephritis. $[3,4]$

Tubular Unresponsiveness to Aldosterone: In certain conditions, namely sickle cell anemia, systemic lupus erythematosus, amyloidosis, and renal transplantation, hyperkalemia may occur due to a defect in the tubular secretion of potassium. This defect has been described due to tubular unresponsiveness to aldosterone, possibly due to a defect in the receptor binding of aldosterone. Plasma renin actively and plasma aldosterone levels are normal. Renal tubules respond to administration of mineralocorticoid by retention of sodium and water but without the increase of K excretion.

\section{Management of Hyperkalemia}

Hyperkalemia especially severe hyperkalemia of $7.9 \mathrm{mmol} / \mathrm{L}$ in the patient presented here is potentially serious and warrants emergency measures. However, an important question is how to validate emergency treatment if the patient is asymptomatic and show no electrocardiographic abnormalities. Nevertheless there is a general tendency among the physicians to become panicky seeing serum $\mathrm{K}$ of $\geq 6.5 \mathrm{mmol} / \mathrm{L}$ and administer calcium gluconate, $50 \%$ dextrose with insulin, and admit the patient into intensive care unit. An important question here is if a patient with severe hyperkalemia has no clinical manifestation and no electrocardiographic abnormalities, is it necessary to infuse them 
with $50 \%$ dextrose solution with insulin and calcium gluconate. There is no data to validate such emergency measure.

Thus the crux of this article is to assert how to decrease serum K level effectively and not necessarily urgently. Since vast majority of the patients with hyperkalemia has chronic renal failure, attention must be focused to find the cause of chronic renal failure and plan treatment to improve renal function which will decrease serum $\mathrm{K}$ to a safe level.

Metabolic acidosis is a concomitant feature of hyperkalemia. Thus if metabolic acidosis is severe for instance Hco3 less than $10 \mathrm{mmol} / \mathrm{L}, 7.5$ percent solution (44.6 MEQ) is available at $50 \mathrm{ml}$ ampoule. Two ampoules of sodium bicarbonate $(100 \mathrm{ml})$ can be given as a direct push then a bicarbonate infusion with $50 \mathrm{mEQ}$ or $100 \mathrm{mEQ}$ sodium bicarbonate in a liter of $5 \%$ dextrose in water (if no diabetes) can be infused at a rate to $60 \mathrm{ml} /$ hour for 48 hours or until serum Hco3 increases $\geq 20 \mathrm{mmol} / \mathrm{L}$. Sodium bicarbonate will combat metabolic acidosis and enhance intracellular translocation of $\mathrm{K}$, thereby reducing serum $\mathrm{K}$ level. It should be noted that sodium bicarbonate infusion is not as helpful for hyperkalemia in dialysis patients as compared to non dialysis patients. Glucose and insulin therapy is very effective for treating hyperkalemia in dialysis patients. Glucose can be given as a 10 percent solution $100 \mathrm{ml}$ per hour for 2 hours along with 10 units of regular insulin for each $10 \mathrm{~g}$ glucose $(100 \mathrm{ml} 10$ percent glucose contains $10 \mathrm{~g}$ glucose)

Albuterol: Adrenergic agonist (albuterol or epinephrine) is very effective in translocating $\mathrm{K}$ rapidly into intracellular space and lowering serum $\mathrm{K}$ level. Albuterol is safer than epinephrine from cardiac standpoint and can be given as 10 to $20 \mathrm{mg}$ by nebulizer or as $0.5 \mathrm{mg}$ intravenously. Although this therapy is effective, but not commonly used.

\section{Sodium Polystyrene Sulfonate}

(Kayexalate $囚$ ). Kayexalate is a powder which is dispensed in $30 \%$ sorbitol and available in $500 \mathrm{ml}$ bottle. For acute hyperkalemia as in the patient reported, usual first dose is $15 \mathrm{~g}$ kayexalate in $60 \mathrm{ml}$ sorbitol then $40 \mathrm{ml}$ every 6 hour until serum $\mathrm{K}+$ is less than $6 \mathrm{mmol} / \mathrm{L}$, then $20 \mathrm{ml}$ three times until serum $\mathrm{K}$ is normal. Kayexalate is dispensed in sorbitol which promotes excretion of $\mathrm{K}+$. Caution must be exercised to prescribe this solution in diabetes patients because sorbitol will increase blood glucose level or may set up new onset diabetes, as well as may aggravate serum $\mathrm{K}$ by releasing $\mathrm{K}$ from the cells. Kayexalate may also be administered as retention enema

In chronic cases of hyperkalemia the one or more following agents may be administered.

1. Kayexalate in sorbitol $5 \mathrm{~g}$ in $20 \mathrm{ml}$ once or twice daily

2. Alpha fluorohydrocortisone (florinef). It is a synthetic aldosterone analog and is very effective in increasing urinary K excretion. Patient must have good urine output to have this drug effective. Usual dose is 0.1 to $0.3 \mathrm{mg}$ per day. First dose can be up to $1 \mathrm{mg}$ in severe hyperkalemia. Side effects include hypertension, edema, and congestive heart failure.

3. Sodium bicarbonate $650 \mathrm{mg}$ PO TID to keep metabolic acidosis under control

\section{Prevention of hyperkalemia}

As stated earlier, acute and chronic renal failure are the most common causes of hyperkalemia. Common causes of chronic renal failure are uncontrolled diabetes, and use of ACEI/ ARB drugs. Therefore use of ACEI/ARB must be avoided in the treatment of diabetes and or hypertension and diabetes must be kept under control with insulin. Patients must strictly follow diet for chronic renal failure avoiding high protein meal and excessive number of fruits to keep K under normal control. Diabetes must be controlled effectively with insulin therapy and not oral antidiabetic agents.

\section{References}

1. Ahuja TS, Freeman D, Manhken JD, Agraharkal M, Siddique M and Menon A. Predictors of the development of hyperkalemia in patients using angiotensin-converting enzyme inhibitors. Am J Nephrol. 2002;20(4):268-272.

2. Mandal AK. A reversible syndrome of acute renal failure associated with renin-angiotensin inhibitor drug. Int J Nephrol U. 2010;2(4):567579 .

3. Arruda JA, Battle Dc, Roseman MK, Barrowski: RL, Kutzman NA and Sehy JT. Hyperkalemia and renal sufficiency: role of selective aldosterone and tubular unresponsiveness. Am J Nephrol. 1981;1(34):160-167.

4. Tan S and Burton M. Hyponerenimic hypoaldosteronism: an overlooked cause of hyperkalemia. Arch Int Med. 1981;141(1):30-33. 